\title{
Assessing Preservation of Tomato with the Use of Microwave Radiation
}

\author{
$\begin{array}{lll}\text { F. G. O. Oni } & \text { O. M. Oni } & \text { P. E. Uhunamure } \\ & & \end{array}$ \\ $1^{1 *}$ Corresponding Author, Department of Crop and Environmental Protection, LAUTECH, Ogbomoso, Nigeria \\ ${ }^{2}$ Department of Pure and Applied Physics \\ ${ }^{1}$ Department of Agronomy, LAUTECH, Ogbomoso, Nigeria
}

\begin{abstract}
Postharvest losses of vegetables can occur at any point from harvest through collection and distribution to the final consumer. Thus, preservation is important for all stake holders involved from planting till consumption of these vegetables. There is therefore the need to look into the use of common methods including home appliances to increase the shelf-life of these produce. Research was conducted to investigate the use of microwave radiation from the kitchen microwave oven for the preservation of fruits using tomato as the test crop. The experiment was conducted inside the Laboratory of Agronomy department, Ladoke Akintola University of Technology LAUTECH, Ogbomoso Oyo state, Nigeria. Tomato fruits were harvested from the teaching and research farm of LAUTECH at maturity stage, and batched into (four) 4 groups of ten (10) tomatoes each then labelled $\left(B_{1}, B_{2}, B_{3}\right.$ and $\mathrm{B}_{0}-$ control). The $\mathrm{B}_{1}, \mathrm{~B}_{2}$, and $\mathrm{B}_{3}$ tomatoes were radiated using the kitchen microwave oven for 5,10 and 15 minutes at three microwave power levels of low $(9.3 \mathrm{GHz})$, med-low $(9.5 \mathrm{GHz})$ and high $(10.0 \mathrm{GHz})$ frequency. The samples were monitored under room temperature for ripening for 5 weeks.The result shows that only $20 \%$ out of the radiated tomatoes at low frequency of $9.3 \mathrm{GHz}$ for 15 minutes were ripened by the $5^{\text {th }}$ week while $\mathrm{B}_{\mathrm{o}}$ tomatoes were all ripened and spoilt by the $3^{\text {rd }}$ week of observation. It is therefore recommended that tomato fruits can be preserved best at a recommended frequency of $9.3 \mathrm{GHz}$ for 15 minutes.
\end{abstract}

Keywords: postharvest losses, Electro-magnetic radiation (EMR), tomato preservation, kitchen microwave oven, DOI: $10.7176 /$ ALST/76-05

Publication date:September $30^{\text {th }} 2019$

\section{INTRODUCTION}

Electro-magnetic radiation (EMR) is a form of radiant energy that travels through a medium and carries that energy with it depositing it into the medium. It can also be said to be a packet of energy propagating through space via proton wave particles which deposits some of its energy on any material that it passes through. In a vacuum, it propagates at a characteristics speed, the speed of light normally in a straight line. As an electromagnetic wave, it has both electric and magnetic field components which oscillates in a fixed relationship to one another, particularly to each other and particularly to the direction of energy and wave propagation (Will and Smith 2008).

Microwave radiation is a form of electromagnetic radiation with wavelength ranging from as long as one meter to as short as one millimeter. The prefix "micro" in "microwave" indicates that microwave is small compared to waves used in typical broadcasting. In that they have shorter wavelength. There are different sources of microwave radiation; the high sources which make use of specialized vacuum tubes to generate microwaves and the lower power sources which makes use of solid state devices such as the field effect transistor. Microwave heats up materials in the process of which energy is being deposited into the source.

Studies on the effect of microwave radiation on plants have been established to be negative. These studies reported that microwave radiation causes burns along the vascular tissues of plant seedlings, damage to the photosynthetic system and significant increase in carotenes and anthocyannins production. The radiation also causes an increase in vegetative reproduction rates of plants growing around base stations (Magone, 1996). However, with the use of sophisticated microwave emitting devices for communications in the air surveillance system, industry, diagnostic and therapeutic purposes in medicine- the importance of electromagnetic pollution need not be over emphasized (Magone, 1996; Markov and Kostarakis, 2007; Valberg. and Van Deventer, 2007 and Akbal, 2010). The evaluation of the health risk from exposure to electromagnetic radiation during microwave cooking is based on measurement of the radiation emission from the appliances. In standardized measurements radiation leakage data were collected from 130 microwave ovens in domestic use, and none of the checked appliances emitted microwave radiation exceeding a power density of $1 \mathrm{MHz} \mathrm{cm}$ at a distance of $5 \mathrm{~cm}$ from the surface of the device. On the basis of statistical calculations, it can be assumed that $50 \%$ of all microwave ovens currently in use emit less than $0.0062 \mathrm{MHz} \mathrm{cm}^{-2}$. If the model of the measurements is valid for all microwave in use, the probability for a single device to exceed the actual emission standard of $5 \mathrm{MHz} \mathrm{cm}^{-2}$ may be less than $0.05 \%$ which indicates that detrimental health effects are not expected to occur as a result of radiation exposure during microwave cooking (Markov and Kostarakis, 2007). Many studies have been undertaken to alleviate fears in the minds of people regarding the deleterious effects, if any, of electromagnetic 
radiation, which has been of late added to the already existing list of environmental pollutants (Oni et al., 2011).

Fruit preservation usually involve preventing the growth of bacteria, fungi (such as yeast), or other microorganisms as well as retarding the oxidation of fats that causes rancidity. Fruit preservation may also include processes that inhibit visual deterioration, such as the enzymatic browning reaction in most fruits and vegetables after they are cut and left for days. Fruits and vegetables can be preserved through different measures such as canning, freezing, drying and heating (Williams, and Tindall 1997; Fayemi, 1999). Heating is one of the most effective ways of preserving fruits and vegetables and this is achieved by placing them in airtight containers and then heat them. Ovens can be used in preservation of fruits by heating using a recommended rate of $9.3 \mathrm{GHz}$ (Skiles and Yoshida, 2006) using the recommended rate ensures that the micro-organisms are killed and the enzymes are inactivated. Any remaining spores will not have the right conditions to grow into bacterial and microbial contamination from outside is prevented. This preservation method produces the best results, but only if fresh products are used and the instructions for heating are followed exactly (Oni et al., 2011).

Tomato which can be referred to as fruit and vegetable, has a higher consumption rate in many developed countries and it is often time referred to as luxury crop. In some countries, it is a part of their diet (Skiles and Yoshida, 2006) and a part the food basket in most developing countries. Farmers are often task with producing quantity and quality and also to produce fruits and vegetables that will stay long even after harvesting. It is worth noting that postharvest losses can occur at any point from harvest through collection and distribution to the final consumer. Thus, preservation is important for all stake holders involved in the production and consumption of these vegetables and fruits.

There is therefore the need to look into the use of common methods of generating heat (which is said to be one of the most effective ways of preserving fruits and vegetables) including home appliances that can be used in increasing the shelf-life of these farm produce. With the increase in use of sophisticated microwave devices such as the kitchen (domestic) microwave oven, this study was to determine if the radiation generated from the home appliance at recommended rate can be used in preserving tomatoes which can also be used for subsequent preservation of similar vegetable crop.

\section{MATERIALS AND METHODS}

\section{Study area}

The experiment was conducted inside the Laboratory of Agronomy department, Ladoke Akintola University of Technology LAUTECH, Ogbomoso Oyo state, Nigeria. The University lies in the derived savannah Agroecological zone characterized by a fairly uniformed annual temperature between $28^{\circ} \mathrm{C}-30^{\circ} \mathrm{C}$. The relative humidity of the area is high (74\%) all year round except in January when the dry wind from the north-east flows in and the annual rainfall of the area is between $1150 \mathrm{~mm}-1250 \mathrm{~mm}$.

\section{Experimental treatment and design}

The experiment was a complete randomized design with a single variety of tomatoes. The Tomato fruits were sourced from the teaching and research farm of LAUTECH to avoid variation in the seed components and eliminate effects of different field managements on the final outcome of the experiment. The fruits were harvested at maturity stage, well before the onset of ripening, sorted and batched into (four) 4 groups of ten (10) tomatoes each then labelled $\left(\mathrm{B}_{1}, \mathrm{~B}_{2}, \mathrm{~B}_{3}\right.$, and $\mathrm{B}_{\mathrm{o}}-$ control). The $\mathrm{B}_{1}, \mathrm{~B}_{2}$ and $\mathrm{B}_{3}$ tomatoes were radiated using the kitchen microwave oven for 5,10 and 15 minutes $\left(T_{1}, T_{2}\right.$, and $\left.T_{3}\right)$ at three microwave power levels of low $(9.3 \mathrm{GHz})$, med-low $(9.5 \mathrm{GHz})$ and high $(10.0 \mathrm{GHz})$ frequency $\left(\mathrm{F}_{1}, \mathrm{~F}_{2}\right.$, and $\left.\mathrm{F}_{3}\right)$ to give a total of ten treatments combinations. The radiated samples and the non-radiated control were placed on labelled trays laid with foil paper under room temperature for ripening and observation was made for 5 weeks, following their ripening stages.

Ripening was recorded as percentage of the total observed tomatoes per batch (10) and compared with control to determine the effectiveness of each radiation frequency and time of exposure on improving the tomato shelf-life.

\section{RESULTS AND DISCUSSION}

Table 1 shows the ripening percentage of tomato at exposure to different radiation from kitchen microwave oven for different frequencies and duration. At the end of first week, ripening was generally low irrespective of the duration and frequency of exposure to the microwave oven radiation except for control and the tomato batch exposed to $10 \mathrm{GHz}$ for 10 minutes with half of each batch $(50 \%)$ ripe while none of the batch exposed to 9.3 $\mathrm{GHz}$ for 15 minutes was ripe. By week two, number of ripe tomato remain same as in week 1 irrespective of the duration and frequency of exposure to the microwave oven radiation except for all $\mathrm{T}_{1}$ batches (ripening increased by $10 \%$ for 9.3 and $9.5 \mathrm{GHz}$ and $20 \%$ for $10 \mathrm{GHz}$ ) and control that has increased to by $30 \%$. As at week three, still no ripening was recorded for the batch exposed to $9.3 \mathrm{GHz}$ for 15 minutes $(0 \%)$, but control had all the tomatoes $(100 \%)$ ripe by then. The percentage recorded at week 4 for the batch exposed to $9.3 \mathrm{GHz}$ for 15 
minutes, was the least $(10 \%)$ while $70 \%$ recorded for the batch exposed to $10 \mathrm{GHz}$ for 10 minutes, was the highest. By week five, $90 \%$ of batch exposed to $10 \mathrm{GHz}$ for 15 minutes are ripe, at least $70 \%$ of batches exposed at 5 minutes are ripe, $10 \mathrm{GHz}$ for 10 minutes are also ripe and the least percentage ripening at week five (20\%) was recorded the batch exposed to $9.3 \mathrm{GHz}$ for 15 minutes.

Table 1: Percentage Number of Ripe Tomato per Week

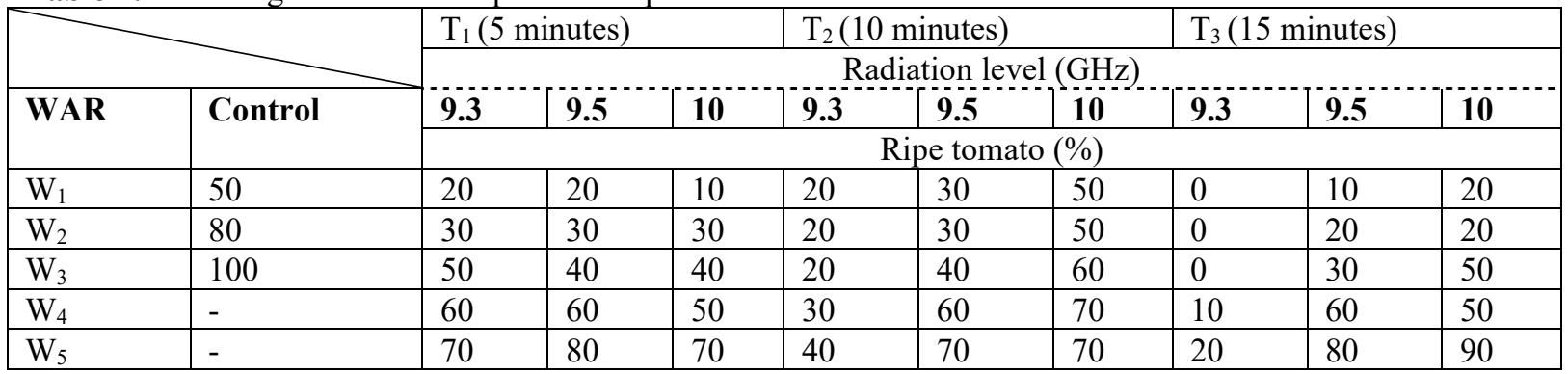

WAR $=$ Weeks After Radiation

Ripening was delayed till the fourth week with exposure of tomatoes to radiation at $9.3 \mathrm{GHz}$ (Fig.1, Fig.2 and Fig.3) which is by far the best of the ten treatments observed in this experiment. Ripening of those tomatoes without radiation was very fast and $100 \%$ were ripe by the third week.

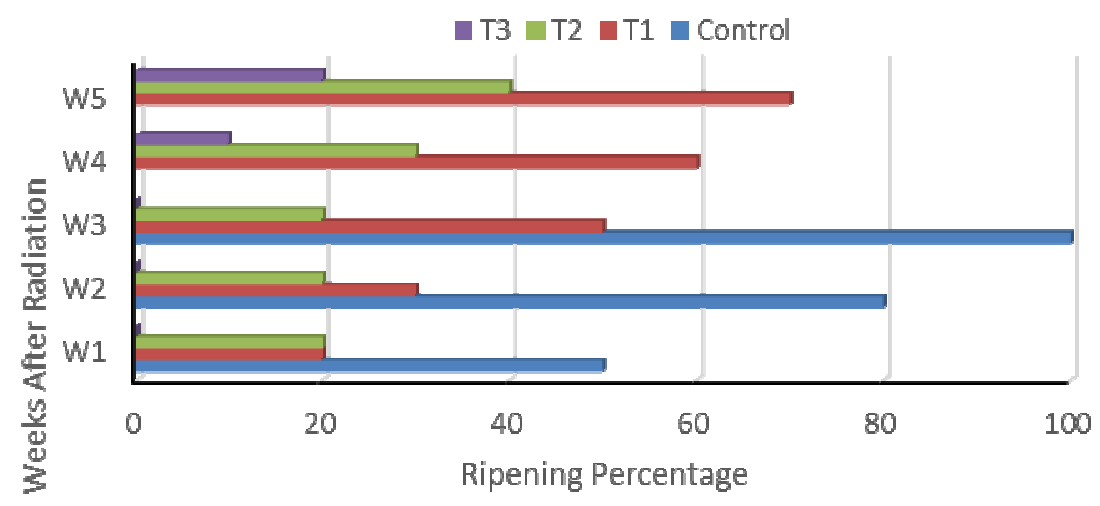

Fig. 1: Ripening rate as affected by radiation of tomato at $9.3 \mathrm{GHz}$

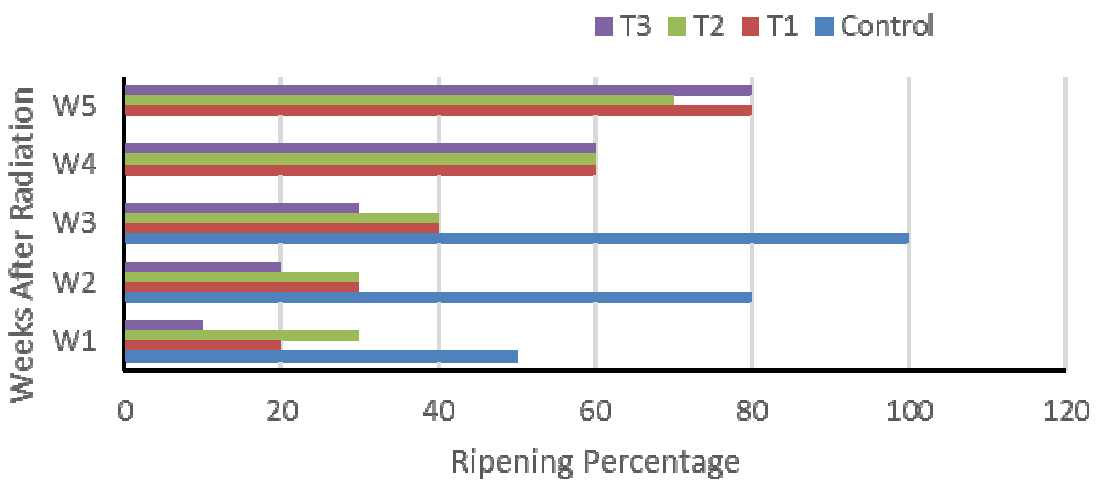

Fig. 2: Ripening rate as affected by radiation of tomato at $9.5 \mathrm{GHz}$ 


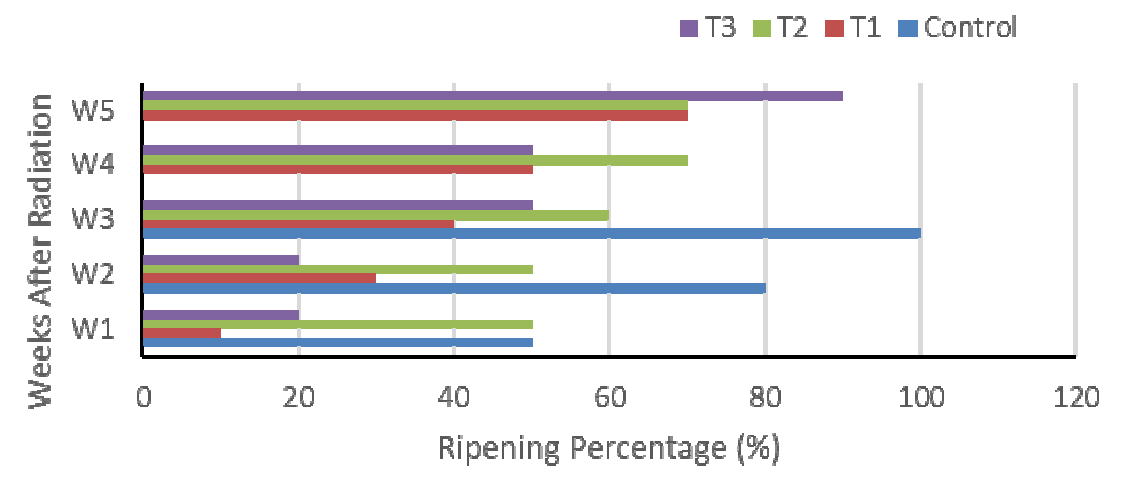

Fig. 3: Ripening rate as affected by radiation of tomato at $10 \mathrm{GHz}$

\section{CONCLUSION AND RECOMMENDATION}

Tomatoes batch exposed to $9.3 \mathrm{GHz}$ at 15 minutes from week 1-week 5 had better shelf-life than others while the control batch had the least. This indicated that for longevity of tomato, preservation at room temperature without any prior postharvest measure, is not advisable. This work also supports earlier findings on effectiveness of heat in vegetable preservation. It is recommended that further investigation should be carried out using higher but safer radiation frequencies and also on other vegetable crops.

\section{REFERENCES}

Akbal, A.A. (2010). Biological effect of electromagnetic field. Environmentalist 27:385

Fayemi, P.O. (1999). Nigeria vegetable, Heinemann education books (PBC), Ibadan pg 50-55.

Magone, I, Tabil. (1996): The effect of electromagnetic radiation from the skrunda radio location station on Spirodela polyrhiza (L.) Sxhleiden cultures, science of the total Environment, 1996, 180, 75-80.

Markov. M. and Kostarakis.P, (2007). Biological effects of electromagnetic fields. Environmentalist 27:385

Oni, O. M, Amuda, D. B., and Gilbert C. E. (2011). Effects of radiofrequency radiation from WiFi devices on human ejaculated semen. Int J Res Rev Appl Sci. 2011;9:292-4

Shirley and Tindall (1997). Fruits and vegetable production in warm climate. The Macmillan press Ltd. Page 85 Skiles, J.W. and Yoshida (2006). Plant response to microwaves, Acta Astronautica, 2006, 58, 258-263

Valberg.P.A, and Van Deventer .E, (2007). wireless network radio frequency (RF) exposure and health consequences. Environ Health Perspective 115(3):416-424

Williams, H. C and Tindall, H.O. (1997). Fruits and vegetable production in warm climate.

Wills H. and Smith. T. (2008). Species of plant parasitic Nematodes associated with forage crops in Eastern Canada Plant Disease Rep. 60:207-210. 